Document downloaded from:

http://hdl.handle.net/10251/147682

This paper must be cited as:

Dhakshinamoorthy, A.; He, J.; Franconetti, A.; Asiri, AM.; Primo Arnau, AM.; García Gómez, $H$. (2018). Defective graphene as a metal-free catalyst for chemoselective olefin hydrogenation by hydrazine. Catalysis Science \& Technology. 8(6):1589-1598. https://doi.org/10.1039/c7cy02404e

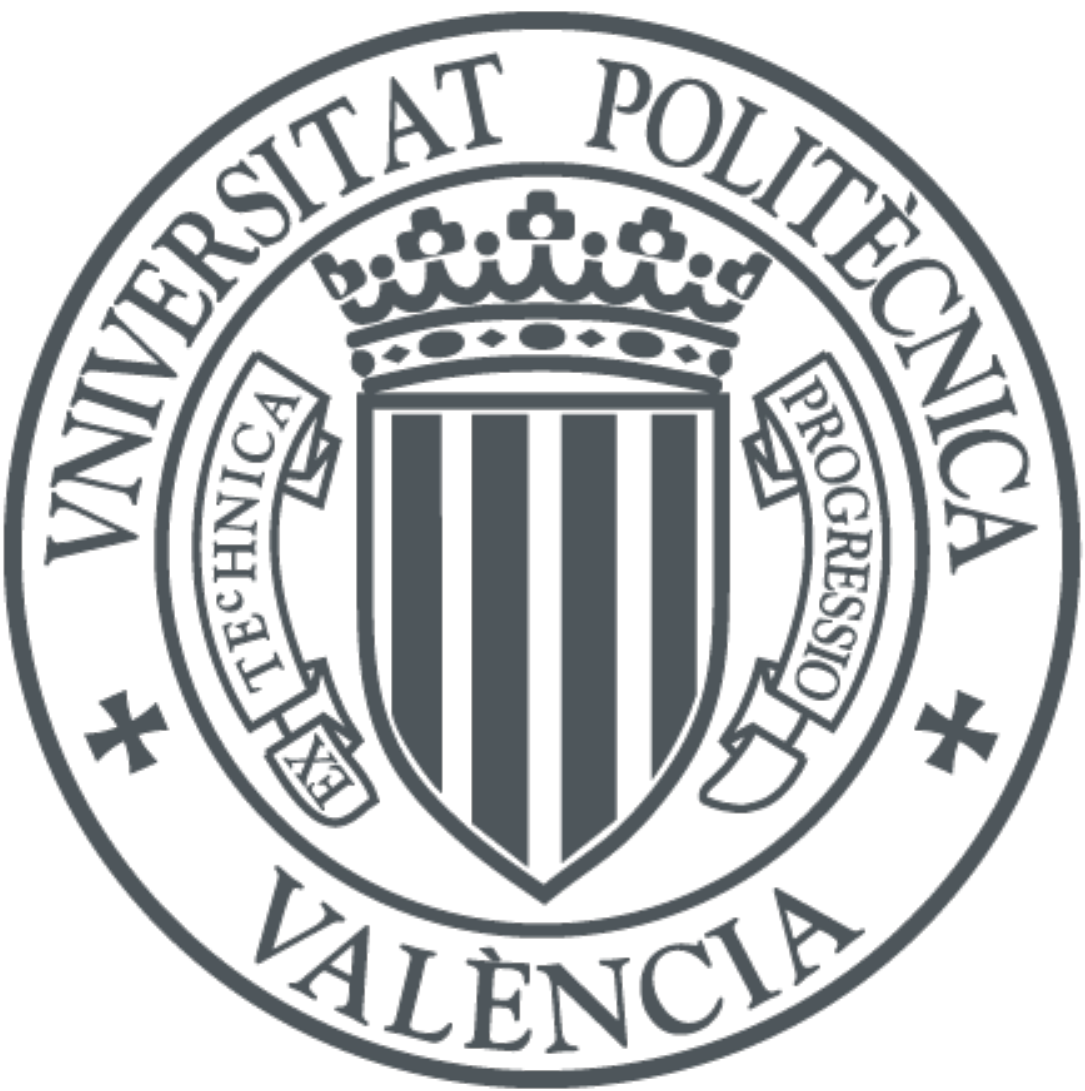

The final publication is available at

https://doi.org/10.1039/c7cy02404e

Copyright The Royal Society of Chemistry

Additional Information 


\section{Defective graphene as metal free catalyst for chemoselective olefin hydrogenation by hydrazine}

Amarajothi Dhakshinamoorthy, ${ }^{\text {a }}$ Jinbao He, ${ }^{\mathrm{b}}$ Antonio Franconetti, ${ }^{\mathrm{c}}$ Abdullah M. Asiri, ${ }^{\mathrm{d}}$ Ana Primo, ${ }^{\text {b Hermenegildo Garcia*b,d }}$

a School of Chemistry, Madurai Kamaraj University, Tamil Nadu, India 625 021.Email:admguru@gmail.com (AD)

b Instituto Universitario de Tecnología Química Consejo Superior de Investigaciones Científicas-Universitat Politécnica de Valencia, Universitat Politècnica de València, Av. De los Naranjos s/n, 46022, Valencia, Spain. E-mail: hgarcia@qim.upv.es (HG)

c Departamento Química Orgánica, Facultad de Química, Universidad de Sevilla, Profesor García Gonzalez 1, 41012 Sevilla (España)

${ }^{\mathrm{d}}$ Centre of Excellence for Advanced Materials Research, King Abdulaziz University, Jeddah, Saudi Arabia.

\section{Abstract}

A series of defective graphenes containing or not $\mathrm{N}, \mathrm{B}, \mathrm{S}$ and other heteroatoms exhibit general activity as metal-free catalysts for the hydrogenation of $\mathrm{C}=\mathrm{C}$ double bonds by hydrazine in the presence of oxygen. The best performing graphene was the one obtained from pyrolysis of alginate and subsequent exfoliation by sonication. The material was reusable in three consecutive runs without decay in its catalytic activity and it exhibits $99 \%$ chemoselectivity for $\mathrm{C}=\mathrm{C}$ double bond vs. nitro group hydrogenation in contrast with conventional Pd supported on carbon that is almost unselective. Theoretical calculations using a model for defective graphene for styrene hydrogenation show adsorption of substrate by $\pi-\pi$ stacking resulting in activation of the double bond and a direct interaction of cisdiimide with the $\mathrm{C}=\mathrm{C}$ group. 


\section{Introduction}

Hydrogenation of multiple bonds is a very important organic reaction which is often catalyzed by expensive homogeneous/heterogeneous metal catalysts including Pd, Pt and Rh. ${ }^{1}$ However, two of the major limitations of this reduction reaction are the high cost of the precious metals used as catalysts and the use of the flammable hydrogen gas. In this context, the search for first-row transition-metal catalysts such as cobalt complex, ${ }^{2}$ arene-cobalt and arene-iron catalyst, ${ }^{3} \mathrm{Fe}$ nanoparticles, ${ }^{4} \mathrm{Fe}$ nanoparticles supported on functionalized graphene, ${ }^{5}$ and $\mathrm{Fe}_{3} \mathrm{O}_{4}$ embedded on graphene oxide (GO) that could act as alternative catalysts of noble metals is an active area of research. ${ }^{6}$ Defective graphenes (Gs) derived from biomass after pyrolysis and exfoliation have been recently reported as metal-free catalysts for hydrogenation nitroaromatics ${ }^{7}$ and selective reduction of alkynes. ${ }^{8}$

Besides the use of molecular hydrogen as reagent, hydrazine hydrate has also been used as hydrogen carrier in the liquid phase reduction of nitroarenes with appropriate catalysts. $^{9-11}$ The use of hydrogen carriers as alternative of hydrogen gas is advantageous in certain cases from the viewpoint of the experimental conditions, since it avoids the use of hazardous, explosive gases and the reaction can be carried out in open reactors.

In view of the above comments, it would be of interest to explore the potential of defective $\mathrm{Gs}$ as $\mathrm{C}=\mathrm{C}$ double bond hydrogenation catalysts using hydrazine as hydrogen carrier. Besides proving the catalytic activity of defective Gs as metal-free catalysts, another issue of interest is to compare its catalytic activity with other carbon allotropes and, particularly, carbon nanotubes (CNTs).

In a series of seminal papers, Su and co-workers reported that single walled CNTs ${ }^{12}$ or B-doped $\mathrm{CNTs}^{13}$ can act as metal free catalysts for hydrogenation of nitro compounds to amines using hydrazine as reducing agent. By means of model compounds, it was proposed 
that the active sites involved in the process are not hydroxyls or carboxylic acids, but keto groups in defective positions. ${ }^{14}$ In comparison with CNTs that can be prepared with low oxygen content due to the composition of the precursors, typically methane or other hydrocarbons, defective Gs obtained by pyrolysis of natural polysaccharides such as alginate or chitosan contain a residual percentage of oxygen from the starting material that is generally in the range of $7-10 \mathrm{wt} \%$ when the process is carried out at $900{ }^{\circ} \mathrm{C} .{ }^{15}$ Therefore, considering that the nature of active sites in single-walled CNTs and Gs are frequently the same, it can be expected that also defective Gs should exhibit catalytic activity for hydrogenation using hydrazine as hydrogen carrier in the presence of oxygen, but with the advantage of having a larger content of oxygenated functional groups and, therefore, presumably higher catalytic activity.

The present manuscript reports the activity of a series of undoped and doped defective Gs obtained from pyrolysis of natural biomass polymers as catalysts for the hydrogenation of $\mathrm{C}=\mathrm{C}$ double bonds by hydrazine in the presence of oxygen, as well as some theoretical calculations on a model to gain information on the reaction mechanism.

\section{Experimental section}

\subsection{Catalysts}

Graphenes used in the present study were prepared by pyrolysis of natural polysaccharides as previously reported (see for the original references). ${ }^{16,17}$

Preparation of GO. Graphite powder (3 g) was suspended in a mixture of concentrated $\mathrm{H}_{2} \mathrm{SO}_{4} / \mathrm{H}_{3} \mathrm{PO}_{4}(360: 40 \mathrm{ml})$ at $0{ }^{\circ} \mathrm{C}$ cooling with an ice bath. Then, $\mathrm{KMnO}_{4}(18 \mathrm{~g})$ was added carefully to this mixture, whereby the occurrence of an exothermic reaction raising the temperature to $35-40{ }^{\circ} \mathrm{C}$ was observed. (Attention: Risk of explosion! Addition of $\mathrm{KMnO}_{4}$ has to be made in lump portions of about $0.5 \mathrm{~g}$ each, particularly at the beginning of the reactions). This reaction mixture was then heated to $50{ }^{\circ} \mathrm{C}$ under stirring for $12 \mathrm{~h}$. The reaction was cooled to room temperature and poured into $400 \mathrm{~g}$ of ice containing $30 \% \mathrm{H}_{2} \mathrm{O}_{2}$ 
(3 ml). The suspension was allowed to cool at room temperature and, then, it was filtered, the solid washed with 1:10 $\mathrm{HCl}(37 \%)$ solution and, then, further with water. The solid was collected and sonicated with $400 \mathrm{ml}$ of water for $30 \mathrm{~min}$ and centrifuged at $4000 \mathrm{rpm}$ for $4 \mathrm{~h}$. The supernatant was centrifuged at $15000 \mathrm{rpm}$ for $1 \mathrm{~h}$. The resulting GO obtained after centrifugation at $15000 \mathrm{rpm}$ was dried at $60^{\circ} \mathrm{C}$.

Preparation of G. Sodium alginate (Sigma, from brown algae) was pyrolized in Ar atmosphere, heating according to the following oven program: $200{ }^{0} \mathrm{C}$ during $2 \mathrm{~h}$ for annealing and, afterwards, heating at $10{ }^{0} \mathrm{C} \min ^{-1}$ up to $900{ }^{0} \mathrm{C}$ for $6 \mathrm{~h}$. This multilayer graphene powder was sonicated at $700 \mathrm{~W}$ for $1 \mathrm{~h}$ to obtain dispersed $\mathrm{G}$.

Preparation of (N)G. Low molecular weight chitosan (Aldrich) was submitted to pyrolysis under $\mathrm{Ar}$ according to the following oven program: $200{ }^{\circ} \mathrm{C}$ for $2 \mathrm{~h}$ to anneal the powder and then heating at $10{ }^{0} \mathrm{C} / \mathrm{min}$ up to $900{ }^{\circ} \mathrm{C}$ for $6 \mathrm{~h}$. The resulting carbonaceous residue was sonicated at $700 \mathrm{~W}$ for $1 \mathrm{~h}$ to obtain dispersed $(\mathrm{N}) \mathrm{G}$. The nitrogen content of the sample was $7.8 \mathrm{wt} \%$, as determined by combustion chemical analysis using a CHNS FISONS elemental analyser.

Preparation of (B)G. $0.5 \mathrm{~g}$ of sodium alginate as powder (Sigma) was dissolved in a boric acid aqueous solution (50 mg of $\mathrm{HBO}_{3}$ in $50 \mathrm{ml}$ of water). The viscous solution was filtered under pressure using syringe filters of $0.45 \mu \mathrm{m}$ of pore diameter to remove solid particles typically present in commercial alginate. The gel was concentrated by water evaporation in an oven at $100{ }^{\circ} \mathrm{C}$ overnight. Pyrolysis was performed under $\mathrm{Ar}$ flow $\left(1 \mathrm{~cm}^{3} \mathrm{~min}^{-1}\right)$ using the following oven program: $200{ }^{\circ} \mathrm{C}$ for $2 \mathrm{~h}$ to anneal the powders and then using heating at 10 ${ }^{0} \mathrm{C} / \mathrm{min}$ up to $900{ }^{0} \mathrm{C}$ for $6 \mathrm{~h}$. The resulting carbonaceous residue was sonicated at $700 \mathrm{~W}$ for $1 \mathrm{~h}$ to obtain dispersed (B)G in water. The boron content was determined by the quantitative analysis provided by XPS giving a value of $2.2 \mathrm{wt} \%$. 
Preparation of $(B, N) G$. Low molecular weight chitosan (Aldrich) (1.00 g) was added into a boric acid aqueous solution (400 $\mathrm{mg}$ of $\mathrm{HBO}_{3}$ in $25 \mathrm{ml}$ of water). An additional amount of acetic acid (0.45 g) was necessary for complete chitosan dissolution. Insoluble solid particles were removed by centrifugation at $6000 \mathrm{rpm}$ during $15 \mathrm{~min}$, removing the impurities present in the bottom. The resulting gel was concentrated by drying in an oven at $100{ }^{\circ} \mathrm{C}$ overnight. Pyrolysis was carried out under Ar flow $\left(1 \mathrm{~cm}^{3} \mathrm{~min}^{-1}\right)$ using the following oven program: $200{ }^{\circ} \mathrm{C}$ for $2 \mathrm{~h}$ to anneal the powder and then heating at $10{ }^{\circ} \mathrm{C} / \mathrm{min}$ up to $900{ }^{\circ} \mathrm{C}$ for $6 \mathrm{~h}$. The resulting carbonaceous residue was sonicated at $700 \mathrm{~W}$ during 1 h to obtain dispersed $(\mathrm{B}, \mathrm{N}) \mathrm{G}$ in aqueous phase. The boron and nitrogen content of the sample was determined by the quantitative analysis provided by XPS giving values of $3.8 \mathrm{wt} \%$ of nitrogen and $3.7 \mathrm{wt} \%$ of boron.

Preparation of (S)G. Commercially available $\lambda$-carrageenan (Sigma Aldrich ref. 22049) was pyrolized under Ar flow $\left(1 \mathrm{~cm}^{3} \mathrm{~min}^{-1}\right)$, first annealing the powder at $200{ }^{\circ} \mathrm{C}$ for $2 \mathrm{~h}$ and, then, heating at $10{ }^{\circ} \mathrm{C} / \mathrm{min}$ up to $900{ }^{\circ} \mathrm{C}$ for $6 \mathrm{~h}$. The resulting graphitic powder was sonicated at $700 \mathrm{~W}$ for $1 \mathrm{~h}$ in water to obtain dispersed (S)G aqueous suspensions. The sulfur content of (S)G was 4.4 wt \% determined by combustion chemical analysis using a CHNS FISONS elemental analyser.

\subsection{Reaction procedure}

In a typical reaction, $20 \mathrm{mg}$ of the catalyst were suspended in $2 \mathrm{~mL}$ of ethanol in a 50 $\mathrm{mL}$ round bottom flask. To this slurry, $1 \mathrm{mmol}$ of styrene and $2 \mathrm{mmol}$ of hydrazine hydrate were added. This reaction mixture was placed in a preheated oil bath maintained at $60{ }^{\circ} \mathrm{C}$ and this reaction mixture was stirred continuously for the required time. A known aliquot of sample was taken periodically to determine the kinetics of the reaction by injecting the samples in gas chromatography. The conversion of styrene was determined by gas chromatography using internal standard and the products were confirmed by GC-MS. 


\subsection{Computational details}

All theoretical calculations were performed by using the Gaussian 09 program package. ${ }^{18}$ Geometry optimizations employed the PBE0 hybrid functional at 6-311G(d,p) level of theory. ${ }^{19,} 20$ During these optimization no symmetry constraints were enforced and the equilibrium structures were verified by absence of imaginary frequencies whereas transition state was calculated by freezing the $\mathrm{N}-\mathrm{H}$ bond of diimide moiety $\left(\mathrm{N}_{\text {img }}=1\right)$. A $3 \times 3$ graphene sheet containing a nitrogen atom and different oxygen atoms such as hydroxyl, carbonyl and carboxyl groups was used as representative model for defective graphene. These models are hydrogen-terminated. However, hydrogen atoms of the edge of graphene models are omitted in the drawings for clarity.

Binding energy ( $\mathrm{kcal} \mathrm{mol}^{-1}$ ) for $\pi-\pi$ non-covalent contacts between graphene and styrene was calculated as follow:

$$
\Delta E_{\text {bind }}=E_{\text {complex }}-E_{\text {graphene }}-E_{\text {styrene }}
$$

in which, $E_{\text {graphene }}$ and $E_{\text {styrene }}$ are the calculated energies at PBE0/6-311G(d,p) level of theory for optimized structures of graphene and styrene, respectively. Following the same strategy, the binding energy for the $\mathrm{NH} / \pi$ was calculated by using the hybrid Minnesota functional M06-2X that is considered to be enough accurate for non-covalent interactions including the dispersion term. ${ }^{21,22}$

\subsection{Results and discussion}

The list of materials under study is presented in Table 1, where the origin and preparation conditions have been indicated as well as some analytical data. As it can be seen in this Table 1, the series of defective Gs includes a $\mathrm{G}$ sample obtained by pyrolysis at $900{ }^{\circ} \mathrm{C}$ of sodium alginate followed by exfoliation by sonication, as well as (N)G and (S)G obtained from chitosan and $\lambda$-carrageenan and containing $\mathrm{N}$ and $\mathrm{S}$, respectively, in addition to $\mathrm{O}$ as co- 
dopant elements. Two boron doped Gs were also prepared by pyrolysis of borate esters of alginate and chitosan followed by sonication of the resulting carbon residues. All these materials have been reported with extensive characterization and spectroscopic data in the literature previously (see Table 1) and the present data from Raman spectroscopy, transmission electron microscopy (TEM), X-ray photoelectron spectroscopy (XPS), chemical analysis and atomic force microscopy (AFM) coincide well with the reported values.

Table 1. List of catalysts used in the present work and their corresponding precursors, preparation method and elemental content. Preparation and detailed characterization of each material is described in the respective reference.

\begin{tabular}{|c|c|c|c|c|c|}
\hline \multirow{2}{*}{ Catalyst } & \multirow{2}{*}{ Precursor } & \multirow{2}{*}{ Preparation method } & \multicolumn{2}{|c|}{ Element content (wt \%) } & \multirow[t]{2}{*}{ Ref. } \\
\hline & & & C & heteroatom & \\
\hline G & Alginate & $\begin{array}{l}\text { Pyrolysis at } 900^{\circ} \mathrm{C} \text {, } \\
\text { exfoliation }\end{array}$ & $64.7^{\mathrm{a}}$ & - & 15 \\
\hline GO & Graphite & $\begin{array}{l}\text { Hummers oxidation, } \\
\text { exfoliation }\end{array}$ & $46.9^{\mathrm{a}}$ & - & 23 \\
\hline (B)-G & $\begin{array}{l}\text { Alginate, } \\
\mathrm{H}_{3} \mathrm{BO}_{3}\end{array}$ & $\begin{array}{l}\text { Pyrolysis at } 900^{\circ} \mathrm{C} \text {, } \\
\text { exfoliation }\end{array}$ & $66.5^{\mathrm{b}}$ & $2.2(\mathrm{~B})^{\mathrm{b}}$ & 16 \\
\hline$(\mathrm{N})-\mathrm{G}$ & Chitosan & $\begin{array}{l}\text { Pyrolysis at } 900^{\circ} \mathrm{C} \text {, } \\
\text { exfoliation }\end{array}$ & $78.5^{\mathrm{a}}$ & $7.8(\mathrm{~N})^{\mathrm{a}}$ & 24 \\
\hline$(\mathrm{B}, \mathrm{N})-\mathrm{G}$ & $\begin{array}{l}\text { Chitosan, } \\
\mathrm{H}_{3} \mathrm{BO}_{3}\end{array}$ & $\begin{array}{l}\text { Pyrolysis at } 900^{\circ} \mathrm{C} \text {, } \\
\text { exfoliation }\end{array}$ & $70.2^{\mathrm{b}}$ & $\begin{array}{l}3.8(\mathrm{~N})^{\mathrm{b}}, \\
3.7(\mathrm{~B})^{\mathrm{b}}\end{array}$ & 25 \\
\hline (S)-G & $\begin{array}{c}\lambda- \\
\text { Carrageenan }\end{array}$ & $\begin{array}{l}\text { Pyrolysis at } 1000^{\circ} \mathrm{C} \text {, } \\
\text { exfoliation }\end{array}$ & $62.0^{\mathrm{a}}$ & $4.4(S)^{\mathrm{a}, \mathrm{c}}$ & 17 \\
\hline
\end{tabular}

${ }^{a}$ The values were determined by combustion chemical analysis; ${ }^{b}$ The values were determined

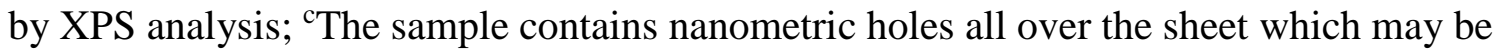
originated by gas evolution during pyrolysis.

Thus, all the samples of Table 1 exhibit in Raman spectroscopy the 2D, G and D bands appearing approximately at 2700, 1600 and $1350 \mathrm{~cm}^{-1}$ that are associated to their few layer packing, the graphenic structure and the presence of defects, respectively. XPS shows by analysis of the C1s peak the existence of graphenic carbons together with carbon bonded to oxygen by single or double bonds, carboxylic acids and carbon bonded to nitrogen or other doped elements. The presence of dopant elements (B, $\mathrm{N}$ and $\mathrm{S}$ ) besides $\mathrm{O}$ was ascertained by 
the observation of the peak corresponding to each element in XPS measurements. Analysis of individual components indicates that there are preferentially two types of nitrogen atoms, either graphenic or pyridinic in about 50:50 proportion, one type of sulphur atom associated to graphenic positions and two types of boron atoms having graphenic or B-O-C-C configuration. The single layer and few layers morphology of the particles was determined by AFM measurements with subnanometric vertical resolution, measuring the thickness of different graphenic platelets. TEM studies show the typical images with light contrast expected for single layer flexible $G$ sheets, exhibiting the characteristic wrinkles, while high resolution TEM and electron diffraction show the hexagonal arrangement of the atoms and the ordering in the sheets. Figs. 1, 2, 3 and 4 show some of the previously commented relevant characterization data that agree well with the reported values (see Table 1 for appropriate references), while the literature contains further exhaustive characterization of the defective $G$ materials included in the present study.

The series of catalysts also includes GO that was obtained from graphite by Hummers oxidation and subsequent exfoliation of graphite oxide by sonication, as reported. ${ }^{23}$ Also, the structure and properties of GO are well documented in the literature. ${ }^{26}$

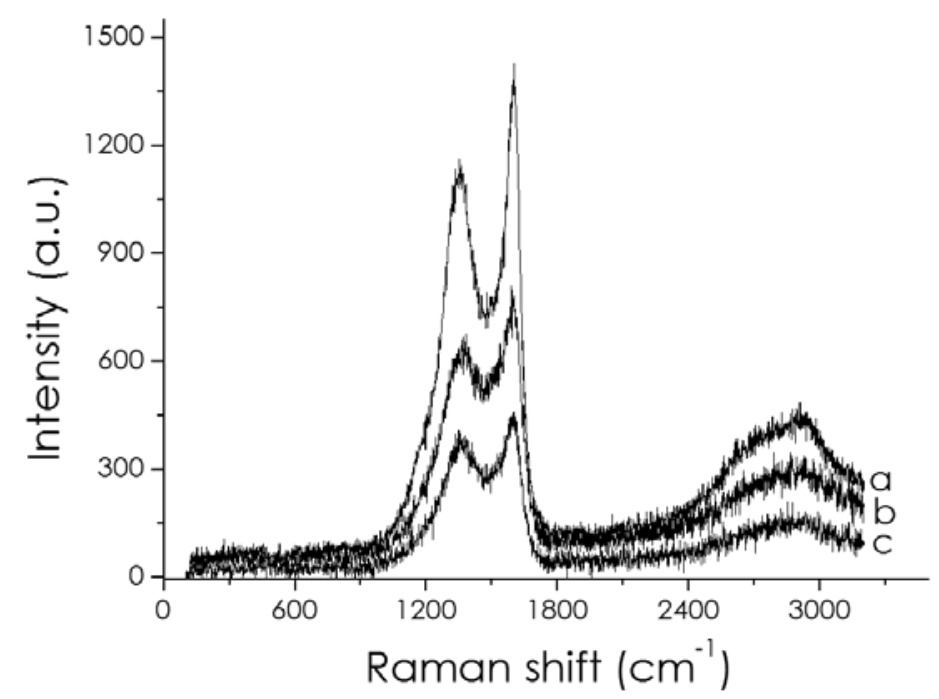

Fig. 1. Raman spectra recorded upon excitation at $613 \mathrm{~nm}$ of $\mathrm{G}(\mathrm{a}),(\mathrm{N}) \mathrm{G}$ (b) and (S)G (c). 

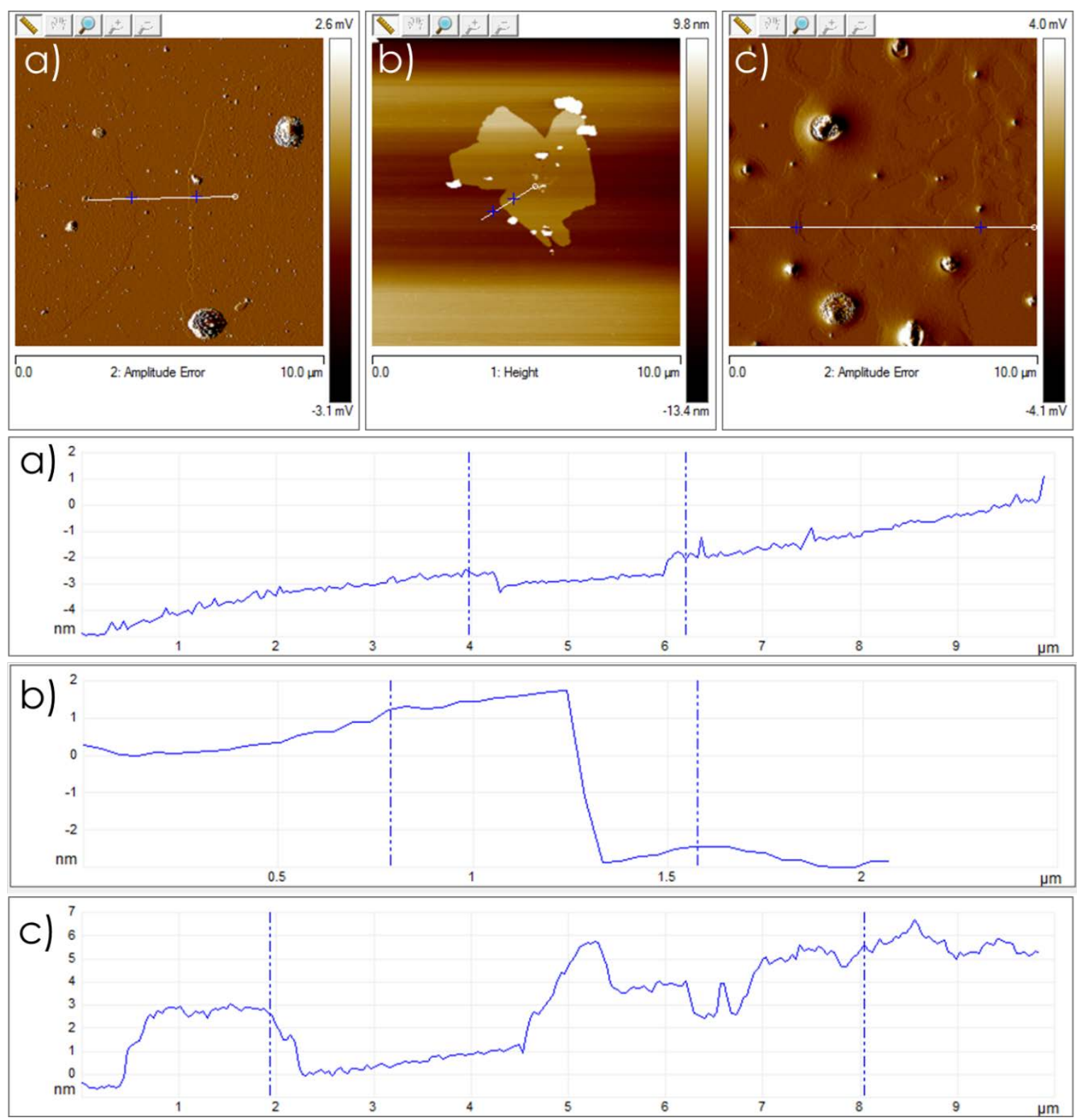

Fig. 2. AFM images of particles of (B)G (a), G (b) and (B,N)G (c) taken by dropping an aqueous dispersion of these graphenes on mica and water evaporation. The bottom panels correspond to height measurements along the white lines indicated in each image. The locations on the top frames indicated as blue crosses are indicated in the vertical height measurements panels with vertical dashed lines. 

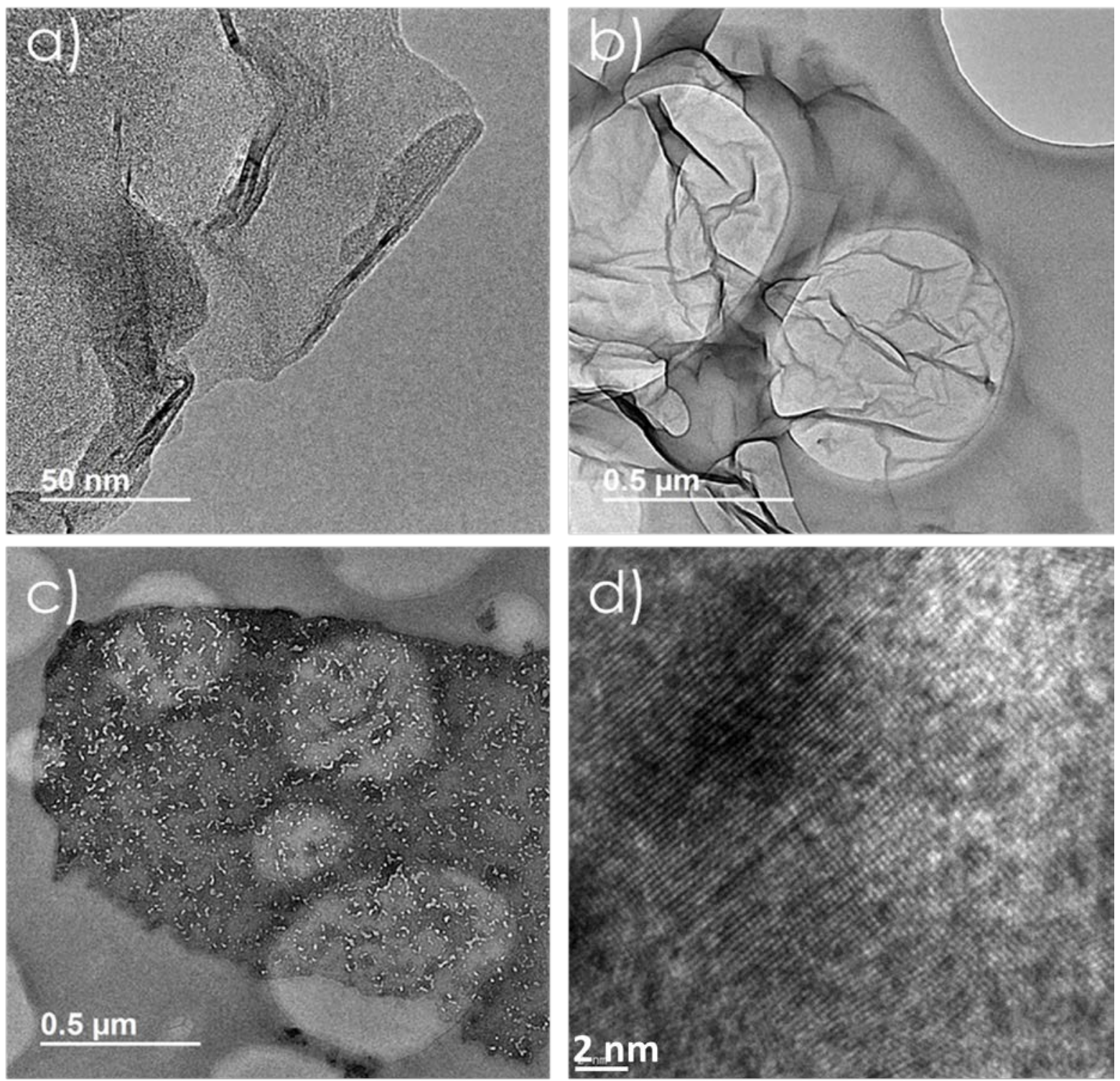

Fig. 3. Representative TEM images at different magnifications of G (a), GO (b), (S)G (c) and (N)G (d). 

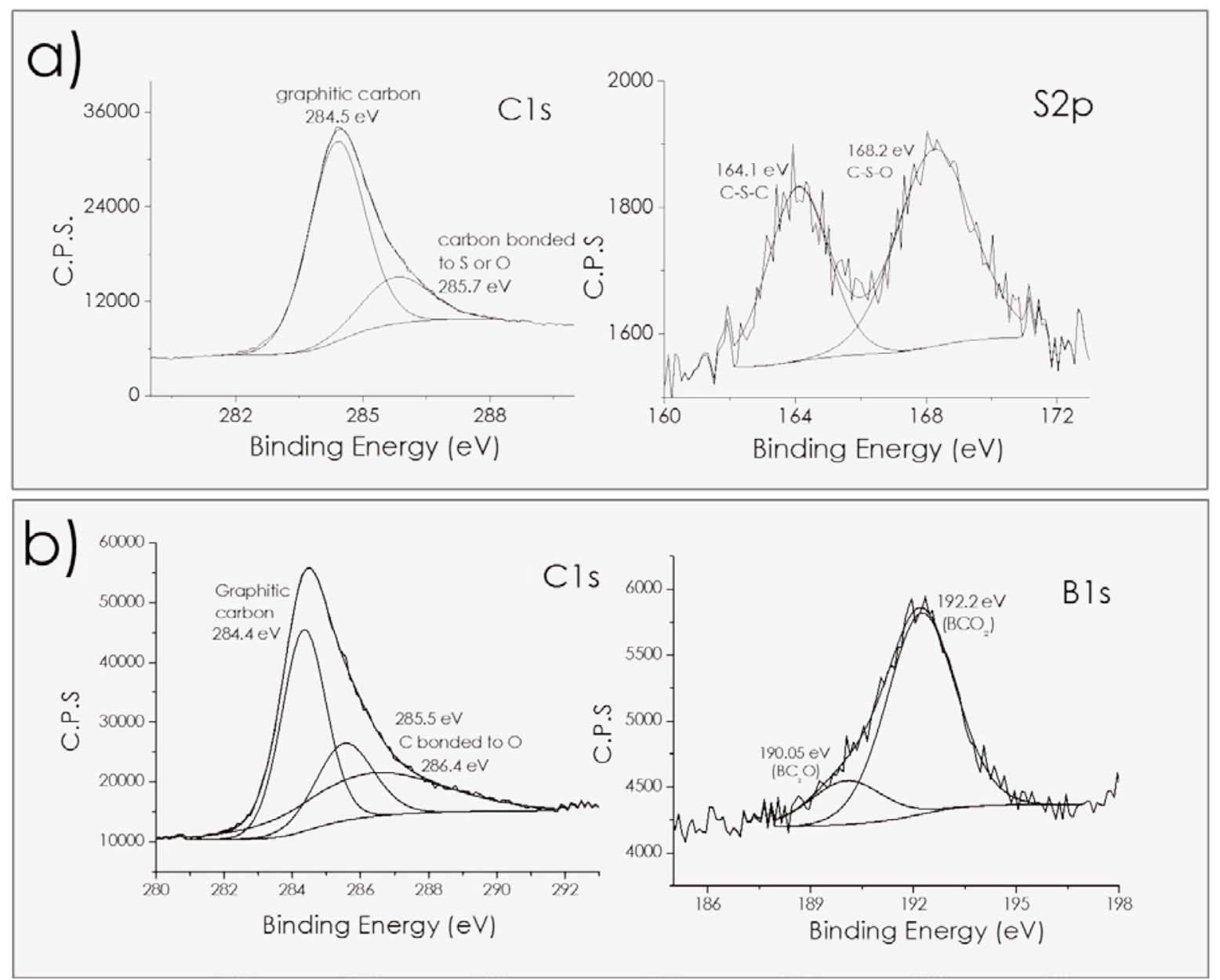

Fig. 4. Experimental XPS peaks and the best fitting to individual components for (S)G (a, C1s and S2p) and (B)G (b, C1s and B1s).

\subsection{Catalytic activity}

In the preliminary stage of our work, the catalytic activity of defective Gs to promote hydrogenation of styrene to ethylbenzene by hydrazine hydrate in ethanol was tested. To check the influence of the presence of oxygen in the process, the reaction was carried out under atmospheric air or oxygen at $60{ }^{\circ} \mathrm{C}$. Fig. 5 shows the time-conversion plot for hydrogenation of styrene under these two reaction conditions. It was observed that the reaction rate in the presence of oxygen is much higher than performing the reaction under air/inert atmosphere, in agreement with the role of $\mathrm{O}_{2} \cdot{ }^{27}$ The presence of oxygen is accordance to reaction mechanism needed to promote the conversion of hydrazine to diimide 
that undergoes spontaneous decomposition into one equivalent of nitrogen and hydrogen and for this reason, the reaction rate under oxygen was much higher than under air/inert atmosphere. ${ }^{27}$ Under our reaction conditions in an oxygen atmosphere, styrene conversion was complete in $8 \mathrm{~h}$. In contrast, a blank control experiment using $\mathrm{G}$ as solid catalyst in the absence of hydrazine provided less than $1 \%$ conversion of styrene after $8 \mathrm{~h}$ at $60{ }^{\circ} \mathrm{C}$ under oxygen atmosphere.

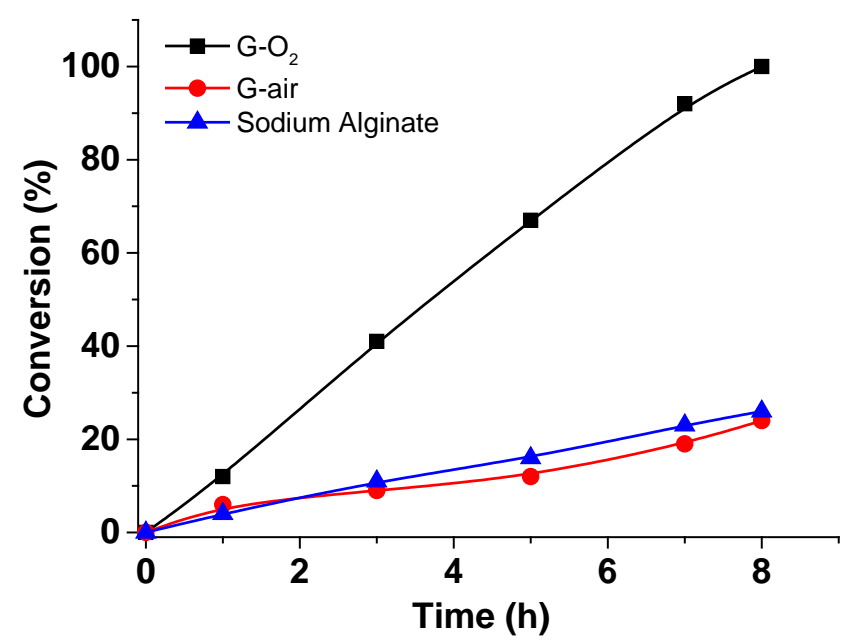

Fig. 5. Time conversion plot for the conversion of styrene to ethylbenzene using defective $G$ with or without oxygen or by sodium alginate. Reaction conditions: styrene (1 mmol), $\mathrm{N}_{2} \mathrm{H}_{4} \cdot \mathrm{H}_{2} \mathrm{O}(2 \mathrm{mmol})$, ethanol $(2 \mathrm{~mL})$, oxygen $\left(\boldsymbol{\square}\right.$ and $\bullet$ ) or air atmosphere $(\boldsymbol{\Delta}), 60{ }^{\circ} \mathrm{C}$. Catalyst. G ( $\mathbf{\square}$ or $\boldsymbol{\Delta})$ or sodium alginate $(\bullet)(20 \mathrm{mg})$.

The catalytic activity of different materials for styrene hydrogenation was checked under optimal reaction conditions. The time-conversion plots for the different graphenes are presented in Fig. 6. It was observed that $(\mathrm{N}) \mathrm{G}$ and $(\mathrm{B}, \mathrm{N}) \mathrm{G}$ are much less efficient catalysts than the other materials of the series. It is proposed that this lower catalytic activity derives from the lower tendency of these N-doped G materials to undergo exfoliation to form a good dispersion in ethanol. Unfortunately, BET surface area measurements based on isothermal nitrogen adsorption on dry powders do not provide information as the surface area of these 
materials in ethanol suspension due to the unavoidable stacking and agglomeration of the $G$ sheets that occurs in dry conditions. On the other hand, the catalytic activity of the rest of the materials was similar, although, defective $G$ derived from sodium alginate was somewhat more efficient than (B)G and (S)G. It is worth noting that the catalytic activity of GO was much higher than that observed for reactions using $\mathrm{H}_{2}$ as reagent and comparable at final reaction time to that of defective G. This is most probably due to the prompt conversion of GO into reduced GO (rGO) that is known to occur upon treatment of GO with hydrazine at room temperature. ${ }^{28}$ In this regard, under the reaction conditions where hydrazine and GO are heated at $60{ }^{\circ} \mathrm{C}$, a fast conversion of GO into rGO should take place at initial stage of the reaction. It should also be commented that rGO and defective G from alginate are similar materials since both contain about 8 wt.\% of residual oxygen and the nature of the oxygenated functionality and other defects are similar in both materials. Therefore, it is not surprising to notice comparable performance of GO (rGO under the reaction conditions) to that of the G material.

It is also worth noting that the fact that (S)G has a similar performance, although somewhat lower, than $G$ indicated that the presence of graphitic sulphur does not influence considerably the activity of the G sheet. This is in agreement with the graphene nature of $\mathrm{S}$ atoms in $(S) G$ and the similar electronegativity of $S$ and $C$ that makes the property of Scontaining heterocycles similar to those of aromatic hydrocarbons.

Considering the previously reported activity of defective Gs as catalysts, ${ }^{29}, 30$ it is proposed that in the present case hydrogenation of styrene also follows the reported reaction mechanism, involving the oxidative transformation of hydrazine to diimide. Subsequently diimide can either react directly with the unsaturated $\mathrm{C}=\mathrm{C}$ double bond ${ }^{31}$ or undergo decomposition to hydrogen that after generation follows the previously reported mechanism 
for hydrogenation of $\mathrm{C}=\mathrm{C}$ bonds by molecular hydrogen involving frustrated Lewis acid base pairs and or carbon vacancies.

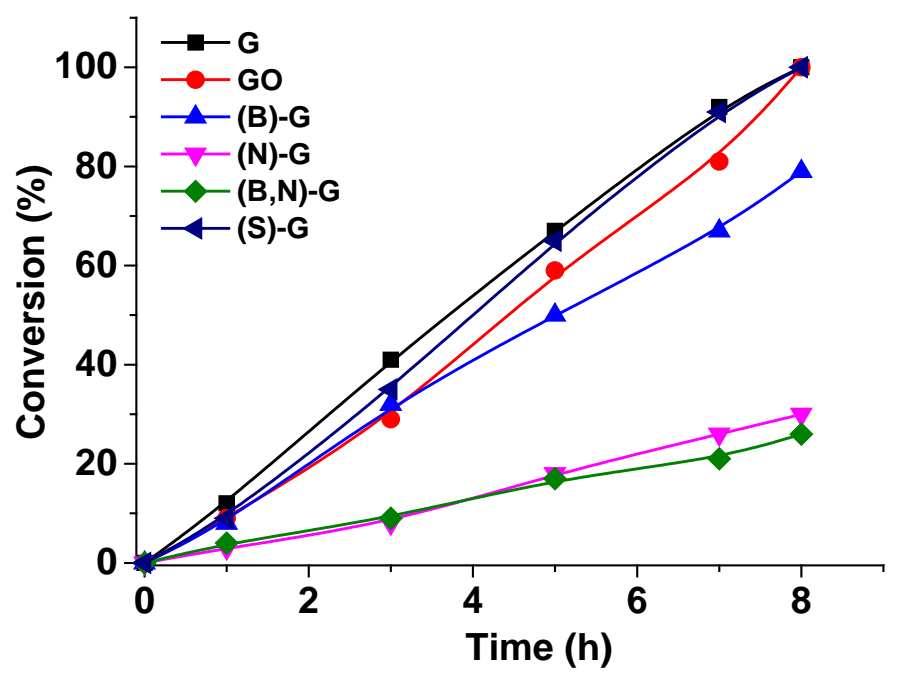

Fig. 6. Comparison of time conversion plots for defective $G$ materials in the reduction of styrene to ethylbenzene. Reaction conditions: styrene ( $1 \mathrm{mmol}$ ), $\mathrm{N}_{2} \mathrm{H}_{4} \cdot \mathrm{H}_{2} \mathrm{O}$ (2 mmol), ethanol (2 mL), oxygen atmosphere, $60{ }^{\circ} \mathrm{C}$, catalyst (20 mg).

One of the advantages of heterogeneous catalysts is their easy recovery from the reaction mixture and the possibility to reuse in consecutive runs. Reusability of the catalyst is related to its stability under the reaction conditions. In this context, defective $G$ was used as catalyst for three consecutive hydrogenation reactions of styrene under the optimized reaction conditions without observing any activity decay, exhibiting identical activity. Fig. 7 presents a plot of styrene conversion in three consecutive reactions. 


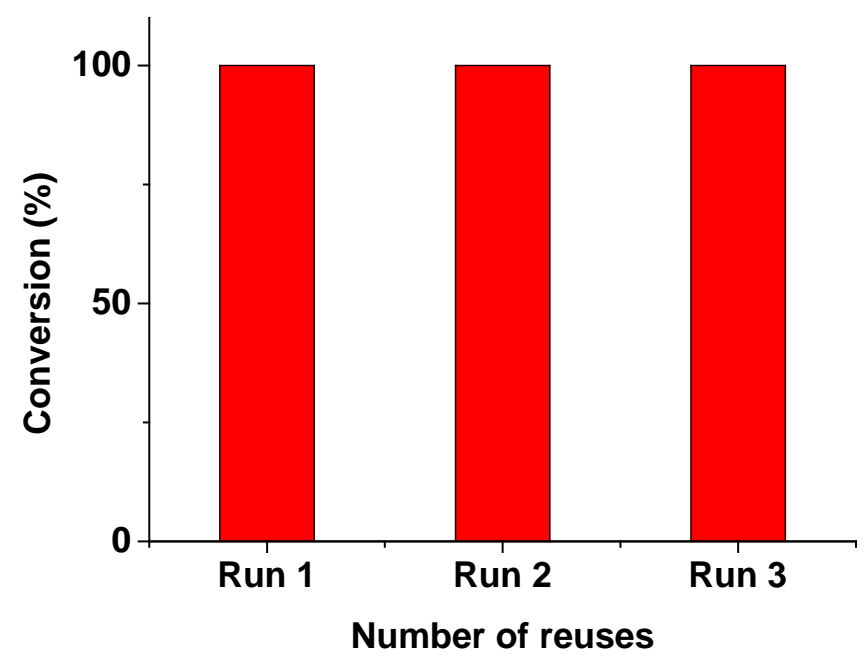

Fig. 7. Reusability of $\mathrm{G}$ for the reduction of styrene to ethylbenzene.

In order to understand the importance of developing carbocatalyst, the performance and chemoselectivity of $\mathrm{G}$ was compared under identical reaction conditions with $10 \mathrm{wt} \%$ $\mathrm{Pd} / \mathrm{C}$ in the hydrogenation of 3-nitrostyrene. This compound has been extensively used as a model substrate to evaluate the activity of $\mathrm{Au}$ catalysts to determine the preferential hydrogenation of nitro groups versus $\mathrm{C}=\mathrm{C}$ double bond. ${ }^{32}$ In the present case, the use of 10 $\mathrm{wt} \% \mathrm{Pd} / \mathrm{C}$ showed the reduction of nitro as well as $\mathrm{C}=\mathrm{C}$ double bond with almost equal selectivity in about $1.5 \mathrm{~h}$ under the reaction conditions. In contrast, the reduction of 3nitrostyrene using $G$ as catalyst exhibited high selectivity with respect to the reduction of $\mathrm{C}=\mathrm{C}$ double bond and nitro group being essentially unaffected, although longer reaction times were required. Scheme 1 compares the catalytic activity between Pd/C and G, showing that the later catalyst exhibits a remarkable chemoselectivity for the hydrogenation of $\mathrm{C}=\mathrm{C}$ double bond in the presence of nitro groups that is not common for transition metal catalysts. 


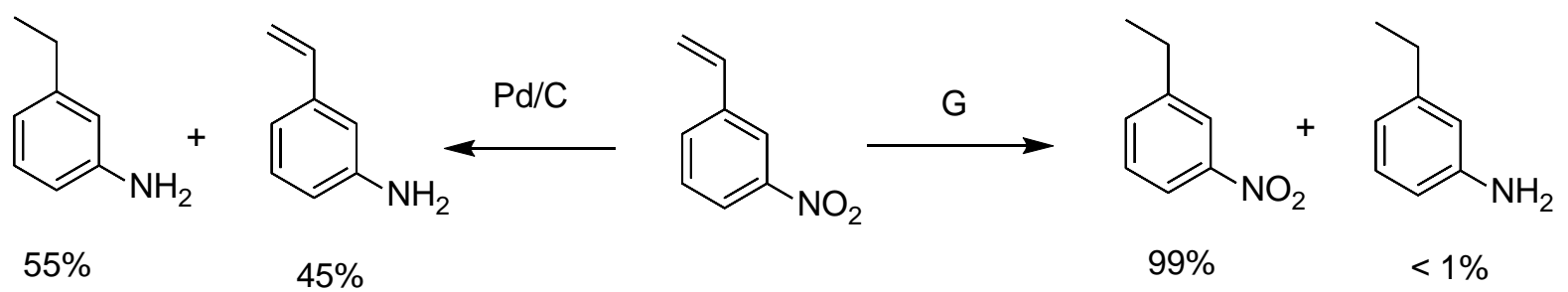

Scheme 1. Comparison of the catalytic activity of $\mathrm{G}$ and $\mathrm{Pd} / \mathrm{C}$ in the reduction of 3nitrostyrene in ethanol at $60{ }^{\circ} \mathrm{C}$. Reaction conditions: 3-nitrostyrene (1 mmol), $\mathrm{N}_{2} \mathrm{H}_{4} \cdot \mathrm{H}_{2} \mathrm{O}(2$ mmol), ethanol (2 mL), oxygen atmosphere, $60{ }^{\circ} \mathrm{C}$, catalyst either $\mathrm{G}(20 \mathrm{mg})$ for $28 \mathrm{~h}$ or $\mathrm{Pd} / \mathrm{C}$ (10 mg) for $1.5 \mathrm{~h}$.

The scope of the catalytic activity of $G$ by hydrazine was screened by studying various substituted styrenes as well as related conjugated $\mathrm{C}=\mathrm{C}$ bonds and allylic, cyclic and acyclic terminal alkenes. The results obtained are summarized in Table 2. As it can be seen there, styrene and their derivatives resulted very high conversion with almost complete selectivity towards their respective reduced products. Sterically hindered styrenes like tanethole and $\alpha$-methylstyrene were converted to their corresponding product in $91 \%$ and 88\% conversions with complete selectivity under the optimized reaction conditions. Furthermore, t-stilbene was smoothly converted to 1,2-diphenylethane in 92\% conversion with complete selectivity. A complete conversion and selectivity was also observed in the reduction of 2-vinylnaphthalene under the present experimental conditions. Similarly, selective hydrogenation relative to hydrogenolysis is a challenging reaction ${ }^{33-35}$ in substrate like allyl phenyl ether. Under the present experimental conditions, this allylic ether was selectively reduced to the corresponding saturated phenyl ether without any cleavage of C-O bond. Furthermore, metal nanoparticles are known to strongly adsorb sulfur-containing molecules and, thus, sulfides are frequently poisons of traditional metal based heterogeneous catalysts. In this aspect, we wanted to check the catalytic activity of $G$ for the reduction of allyl phenyl sulphide under the present experimental conditions. To our delight, the allyl sulfide was hydrogenated to the corresponding reduced product in $94 \%$ conversion with 
complete selectivity. On the other hand, vinylcyclooctane, cis-cyclooctene and 1-decene were also hydrogenated to their respective products in high conversion and selectivity. An attempt to reduce 1-phenylcyclohexene showed $14 \%$ conversion after $48 \mathrm{~h}$ and this low activity can be probably due to steric encumbrance around tertiary cyclic alkene. Also, Nvinylcaprolactam in where a terminal alkene is connected to the amide nitrogen atom required longer time for complete conversion (entry 16, Table 2), probably due to low electron density of this $\mathrm{C}=\mathrm{C}$ bond, having electron withdrawing substituents. In contrast, most of the conjugated $\mathrm{C}=\mathrm{C}$ double bonds gave high conversions under the present reaction conditions.

Table 2. Reduction of olefins to their corresponding alkanes using defective Gs as carbocatalyst. $^{\mathrm{a}}$

\begin{tabular}{|l|l|l|l|l|}
\hline Run & Substrate & $\begin{array}{l}\text { Time } \\
(\mathrm{h})\end{array}$ & $\begin{array}{l}\text { Conversion } \\
(\%)^{\mathrm{b}}\end{array}$ & $\begin{array}{l}\text { Selectivity } \\
(\%)^{\mathrm{b}}\end{array}$ \\
\hline 1 & Styrene & 7 & 100 & 100 \\
\hline 2 & 4-Chlorostyrene & 17 & 100 & 100 \\
\hline 3 & 4-Methoxystyrene & 18 & 100 & 100 \\
\hline 4 & 4-Fluorostyrene & 24 & 88 & 100 \\
\hline 5 & 3-Nitrostyrene & 28 & 98 & 99 \\
\hline 6 & t-Anethole & 24 & 91 & 100 \\
\hline 7 & $\alpha-M e t h y l s t y r e n e$ & 24 & 88 & 100 \\
\hline 8 & t-Stilbene & 24 & 92 & 100 \\
\hline 9 & 2-Vinylnaphthalene & 7 & 100 & 100 \\
\hline 10 & Allyl phenyl ether & 28 & 82 & 100 \\
\hline 11 & Allyl phenyl sulphide & 30 & 94 & 100 \\
\hline 12 & Vinylcyclooctane & 20 & 100 & 100 \\
\hline 13 & Cis-cyclooctene & 24 & 76 & 100 \\
\hline 14 & 1-decene & 16 & 100 & 100 \\
\hline 15 & 1-phenylcyclohexene & 48 & 14 & 100 \\
\hline 16 & N-Vinylcaprolactam & 24 & 62 & 100 \\
\hline
\end{tabular}


aReaction conditions: olefin ( $1 \mathrm{mmol}), \mathrm{N}_{2} \mathrm{H}_{4} \cdot \mathrm{H}_{2} \mathrm{O}$ (2 mmol), G (20 mg), ethanol (2 mL), oxygen atmosphere, $60{ }^{\circ} \mathrm{C}$. b Determined by GC.

Theoretical calculations based on a $3 \times 3$ graphene sheet model were applied to understand the catalytic activity of $G$ as free metal catalyst. The optimized structure of this model (Fig. 8) shows a completely planar structure with a $\mathrm{C}\left(\mathrm{sp}^{2}\right)-\mathrm{C}\left(\mathrm{sp}^{2}\right)$ bond length about $1.42 \AA$ A. The model was implemented with hydroxyl, carbonyl and carboxyl groups that should be present on the graphene sheet as consequence of the residual oxygen content in defective graphenes obtained from pyrolysis of polysaccharides. Moreover, these functionalities together with the pyridine moiety induce an average on the $\mathrm{C}\left(\mathrm{sp}^{2}\right)-\mathrm{C}\left(\mathrm{sp}^{2}\right)$ bond length between $1.33 \AA$ and $1.54 \AA$.

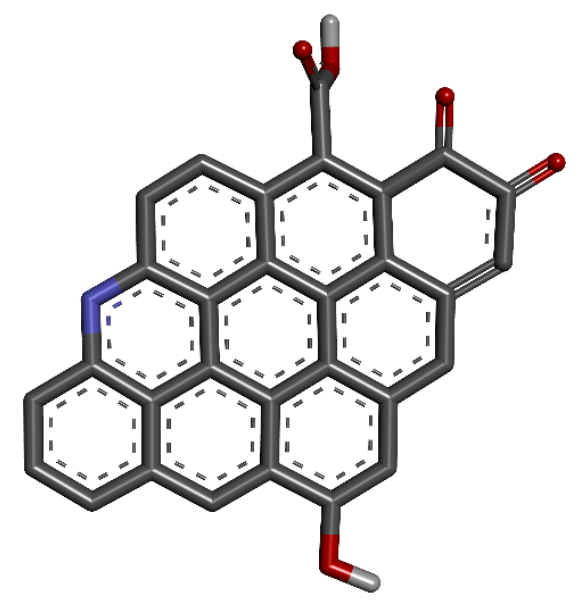

Fig. 8. The $3 \times 3$ graphene sheet model optimized at PBE0/6-311G(d,p) level of theory. Color code: carbon is grey, oxygen is red, hydrogen is white, and nitrogen is blue. Non-polar hydrogen atoms at the periphery of the G model are omitted.

This catalyst allows the stabilization of styrene and styrene-derived alkenes through the widely described $\pi-\pi$ interaction. ${ }^{36}$ Thus, after the optimization styrene was located on the catalyst surface close to the oxidized edge. The stabilization of the catalyst $\cdots$ styrene system $\left(\Delta E_{\text {bind }}=-5.1 \mathrm{kcal} \mathrm{mol}^{-1}\right)$ leads a $\pi$-stacked structure with an equilibrium distance of $3.58 \AA$ (Fig. 9). In addition, this stabilization provokes a change on the Mulliken charge distribution 
of styrene being significant for the $\mathrm{C}=\mathrm{C}$ bond (i.e. from -0.21 to -0.25 for methylene carbon), thus favoring the reduction process.

(a)

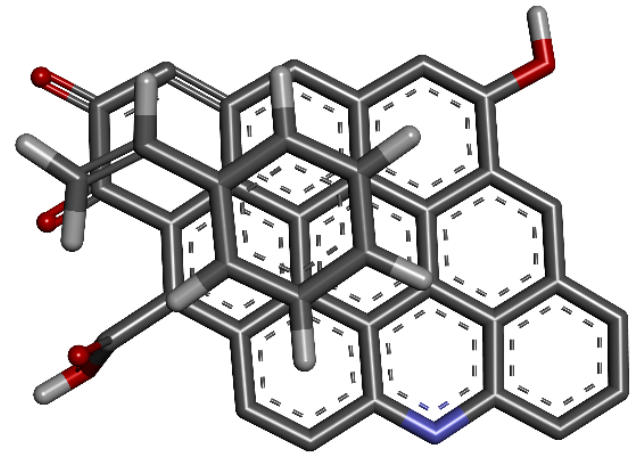

(b)

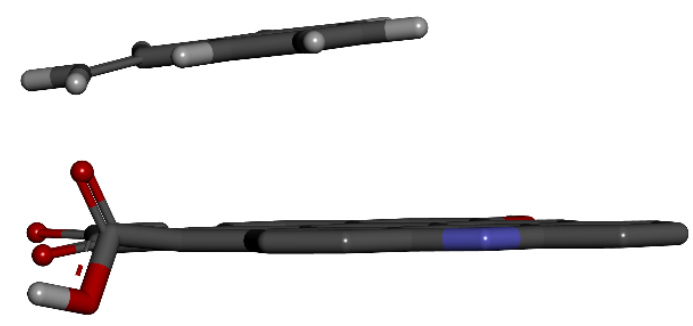

Fig. 9. Frontal (a) and lateral (b) views of the results of the calculations at PBE0/6-311G(d,p) level of theory for the interaction of styrene and the defective graphene sheet model showing the $\pi-\pi$ interaction. Color code: carbon is grey, oxygen is red, hydrogen is white, and nitrogen is blue. Non-polar hydrogen atoms located at the periphery of of G are omitted.

Diimide intermediate is generated from oxidation of $\mathrm{N}_{2} \mathrm{H}_{4} \cdot \mathrm{H}_{2} \mathrm{O}$ in presence of vicinal carbonyl groups on the edge of graphene. ${ }^{37}$ In this situation, vicinal carbonyl groups end up in their reduced form (hydroxyl group) affording the aromatic catechol-like substructures as well as a cis diimide intermediate. The theoretical outcomes reveal that this diimide intermediate is firstly stabilized on the top of $\mathrm{C}=\mathrm{C}$ bond strongly modifying the charge distribution of styrene (i.e. from -0.25 to -0.35 for methylene carbon). In fact, even transconfigured diimide intermediate was isomerized to cis intermediate to maximize the interaction in which the corresponding $\mathrm{N}-\mathrm{H}$ protons are pointing towards $\mathrm{C}=\mathrm{C}$ bond of styrene moiety $\left(R_{\mathrm{C} \cdots \mathrm{HN}}=2.09\right.$ and $2.16 \AA$, respectively). The interaction with the diimide moiety slightly modify the distance styrene $\cdots$ graphene from 3.58 to $3.62 \AA$ that are still, however, in the length range expected for $\pi-\pi$ contacts. This ternary system, less stable than the final product, proved to be an initial pre-stabilization before the final conversion to the products (ethylbenzene and $\mathrm{N}_{2}$ ). Further insights on the origin of styrene $\cdots$ diimide interaction 
suggest two kind of $\mathrm{NH} / \pi$ contacts with a calculated $\Delta E_{\text {bind }}$ of $-6.9 \mathrm{kcal} \mathrm{mol}^{-1}$ at M06-2X/631G(d,p) level of theory.

The energy profile for adsorption of styrene and cis-diimide on the graphene sheet model, the corresponding transition state (TS) and the hydrogenation reaction are presented in Fig. 10. Energy calculations show that adsorption of the reagents on the graphene model results in a stabilization of about $0.5 \mathrm{eV}$ due to the appearance of $\pi-\pi$ interaction and $\mathrm{NH} / \pi$ non-covalent contacts. The calculation predicts a final stabilization of $-3.63 \mathrm{eV}$ after the complete conversion of styrene to ethylbenzene (and $\mathrm{N}_{2}$ ).

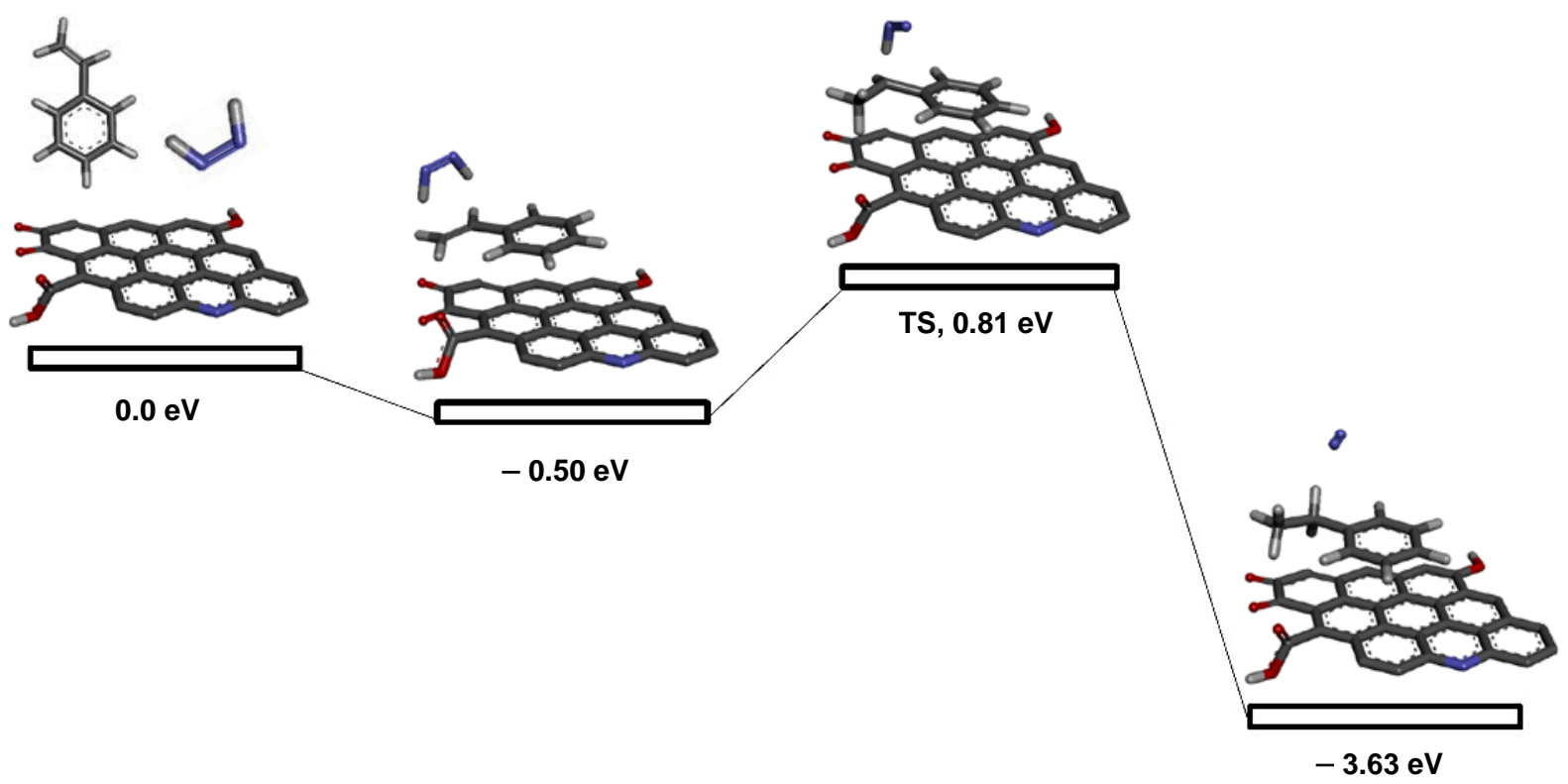

Fig. 10. Optimized minima $\left(\mathrm{N}_{\mathrm{imag}}=0\right)$ and energy profile $(\mathrm{eV})$ for the ternary systems involved in the hydrogenation of styrene. Color code: carbon is grey, oxygen is red, hydrogen is white, and nitrogen is blue. Non-polar hydrogen atoms of G are omitted.

\section{Conclusions}

In the present manuscript, it has been shown that, similarly to the case reported for SWCNTs, also defective Gs exhibit general catalytic activity for hydrogenation of $\mathrm{C}=\mathrm{C}$ double bonds by hydrazine as reducing agent. Among the series of doped and undoped Gs, the sample that exhibits the highest catalytic activity was, the material obtained by pyrolysis of sodium alginate, affording a defective $\mathrm{G}$ having a residual oxygen content of around $9 \%$. 
This material exhibits a general activity for hydrogenation of substituted styrenes, conjugated $\mathrm{C}=\mathrm{C}$ double bonds, acyclic terminal alkenes, decreasing the activity for encumbered trisubstituted cyclic alkenes. Calculations based on a model of defective graphene as well as catalytic data on the influence of oxygen support that the active hydrogen species is cisdiimide that transfer hydrogen to alkene adsorbed on the graphene sheet. Possible sites for hydrazine conversion could be ketone groups at defective centres that promote the oxidation to diimide.

\section{Acknowledgements}

AD thanks the University Grants Commission (UGC), New Delhi, for the award of an Assistant Professorship under its Faculty Recharge Programme. AD also thanks the Department of Science and Technology, India, for the financial support through Extra Mural Research Funding (EMR/2016/006500). Financial support by the Spanish Ministry of Economy and Competitiveness (Severo Ochoa SEV2016-0683, Grapas and CTQ2015-69563CO2-1) and Generalitat Valenciana (Prometeo) is gratefully acknowledged.

\section{Conflicts of Interest}

There are no conflicts of interest

\section{References}

1. J. G. de Vries and C. J. Elsevier, Handbook of homogeneous hydrogenations, Wiley-VCH, New York, 2007.

2. S. Monfette, Z. R. Turner, S. P. Semproni and P. J. Chirik, J. Am. Chem. Soc., 2012, 134, 45614564.

3. D. Gartner, A. Welther, B. R. Rad, R. Wolf and A. Jacobi von Wangelin, Angew. Chem., Int. Ed., 2014, 53, 3722-3726.

4. R. Hudson, G. Hamasaka, T. Osako, Y. M. A. Yamada, C.-J. Li, Y. Uozumi and A. Moores, Green Chem., 2013, 15, 2141-2148.

5. M. Stein, J. Wieland, P. Steurer, F. Tclle, R. Mulhaupt and B. Breit, Adv. Synth. Catal., 2011, 353, 523-527.

6. J. Mondal, K. T. Nguyen, A. Jana, K. Kurniawan, P. Borah, Y. Zhao and A. Bhaumik, Chem. Commun., 2014, 50, 12095-12097.

7. M.-M. Trandafir, M. Florea, F. Neatu, A. Primo, V. I. Parvulescu and H. Garcia, ChemSusChem, 2016, 9, 1565-1569. 
8. A. Primo, F. Neatu, M. Florea, V. Parvulescu and H. Garcia, Nat. Commun., 2014, 5, 52915299.

9. A. Furst, R. C. Berlo and S. Hooton, Chem. Rev., 1965, 65 51-68.

10. Q. Shi, R. Lu, L. Lu, X. Fu and D. Zhao, Adv. Synth. Catal., 2007, 349, 1877-1881.

11. F. Li, B. Frett and H. Y. Li, Synlett 2014, 1403-1408.

12. S. Wu, G. Weng, R. Schlogl and D. S. Su, Phys. Chem. Chem. Phys., 2015, 17, 1567-1570.

13. Y. Lin, S. Wu, W. Shi, B. Zhang, J. Wang, Y. A. Kim, M. Endo and D. S. Su, Chem. Commun., 2015, 51, 13086-13089.

14. S. Wu, G. Wen, X. Liu, B. Zhong and D. S. Su, ChemCatChem, 2014, 6, 1558-1561.

15. A. Primo, A. Forneli, A. Corma and H. García, ChemSusChem, 2012, 5, 2207-2214.

16. A. Dhakshinamoorthy, A. Primo, P. Concepcion, M. Alvaro and H. Garcia, Chem. - Eur. J., $2013,19,7547-7554$.

17. A. Dhakshinamoorthy, M. Latorre-Sanchez, A. M. Asiri, A. Primo and H. Garcia, Catal. Commun., 2015, 65, 10-13.

18. n. 1, Journal.

19. C. Adamo and V. Barone, The Journal of Chemical Physics, 1999, 110, 6158-6170.

20. M. Ernzerhof and G. E. Scuseria, The Journal of Chemical Physics, 1999, 110, 5029-5036.

21. Y. Zhao and D. G. Truhlar, Accounts of Chemical Research, 2008, 41, 157-167.

22. Y. Zhao, N. E. Schultz and D. G. Truhlar, Journal of Chemical Theory and Computation, 2006, 2, 364-382.

23. W. S. Hummers and R. E. Offeman, J. Am. Chem. Soc., 1958, 80, 1339-1339.

24. A. Primo, P. Atienzar, E. Sanchez, J. M. Delgado and H. Garcia, Chem.Commun. , 2012, 48, 9254-9256.

25. I. Esteve-Adell, B. Crapart, A. Primo, P. Serp and H. Garcia, Green Chem., 2017, 19, 30613068.

26. D. R. Dreyer, S. Park, C. W. Bielawski and R. S. Ruoff, Chem. Soc. Rev. , 2010, 39, 228-240.

27. A. T. Murray and Y. Surendranath, ACS Catal. , 2017, 7, 3307-3312.

28. C. Su and K. P. Loh, Acc. Chem. Res., 2013, 46 2275-2285.

29. D. Deng, K. S. Novoselov, Q. Fu, N. Zheng, Z. Tian and X. Bao, Nature Nanotechnol., 2016, 11, 218-230

30. S. Navalon, A. Dhakshinamoorthy, M. Alvaro, M. Antonietti and H. Garcia, Chem. Soc. Rev., 2017, 46, 4501-4529.

31. D. J. Pasto, R. T. Taylor, D. J. Pasto and R. T. Taylor, Organic Reactions; John Wiley \& Sons, Inc.: Hoboken, NJ, USA, 1991; pp 91-155.

32. P. Serna and A. Corma, ACS Catal., 2015, 5, 7114-7121.

33. S. Banerjee, V. Balasanthiran, R. T. Koodali and G. A. Sereda, Org. Biomol. Chem., 2010, 8, 4316-4321.

34. Y. Monguchi, T. Marumoto, T. Ichikawa, Y. Miyake, Y. Nagae, M. Yoshida, Y. Oumi, Y. Sawama and H. Sajiki, ChemCatChem, 2015, 7, 2155-2160.

35. A. Perosa, P. Tundo and S. Zinovyev, Green Chem., 2002, 4, 492-494.

36. L. M. Salonen, M. Ellermann and F. Diederich, Angewandte Chemie International Edition, 2011, 50, 4808-4842.

37. W. Ratnayake, J. Grossert and R. Ackman, Journal of the American Oil Chemists' Society, 1990, 67, 940-946. 